\title{
Magneto-Optical Tools to Study Effects in Dirac and Weyl Semimetals
}

\author{
Dima Cheskis
}

Physics Department, Faculty of Natural Sciences, Ariel University, 40700 Ariel, Israel; dimach@ariel.ac.il; Tel.: +972-3-6453-128

Received: 30 June 2020; Accepted: 23 August 2020; Published: 25 August 2020

\begin{abstract}
Research regarding topological Dirac and Weyl semimetals contributes to our understanding not only of the field of solid-state physics, but also the field of high-energy physics as the physics of Dirac and Weyl semimetals resembles the physics of Dirac and Weyl massless fermions. In condensed matter physics, the Weyl nodes are detached in momentum space and may be realized as emergent quasiparticles with a distinct chirality, left-handed or right-handed. These states lead to phenomena like the chiral anomaly and the anomalous Hall effect (AHE). Furthermore, the combination of quantum effects and magnetic effects in magnetic Weyl semimetals is very intriguing. Magneto-optical tools, which are usually used to study magnetic phenomena, also contribute to magnetic Weyl semimetals. Moreover, with the magneto-optical technique, it is possible to follow the dynamics of the processes and to study the lifetime of the Weyl states. In this work, we review and discuss the effects of using magneto-optical tools for studying quantum effects like the chiral anomaly or magnetic effects in magnetic Weyl and Dirac systems using the magneto-optical Kerr effect (MOKE) or Faraday systems including a single detection and imaging. Examples of using magneto-optical systems in the research of ultrafast magnetic dynamics of thin polycrystalline nickel and permaloy are reviewed as are the magnetic spatial dynamics by employing magneto-optical Kerr or Faraday microscopy tools with ferromagnetic thin films. Interestingly, the excitation of a circularly polarized femtosecond laser pulse could lead to the breakage of time-reversal symmetry and to the transformation of the Dirac state to the Floquet-Weyl semimetal state. The development of a suitable ultrafast magneto-optical system for Weyl systems is discussed, and the practical difficulties for the realization of such a system are considered.
\end{abstract}

Keywords: Weyl semimetal; graphene; chiral anomaly

\section{Introduction}

In recent years, the physics community has taken up a new and exciting challenge: conducting high-energy experiments in condensed matter systems. Such experiments can reveal new properties of the medium and test the limits of theoretical models. For example, theoretical, solid-state models of graphene [1] invoke the Dirac equation, which also arises in cosmology and accelerator physics. Since the characterization of graphene, many other materials with nodal band crossings have been found. These materials are now called topological insulators or semimetals [2-9]. Topological Dirac [10-12] and Weyl [13] semimetals are named after the Dirac [14] and Weyl [15] fermions in high-energy physics. Although these are both solid materials with crystalline structures, low-energy electron excitations in the materials behave similarly to the respective fermions.

Interestingly, while the Weyl fermion plays a major role in the standard model [15], it has never been detected as a fundamental particle in experiments. One of its properties is the so-called chiral anomaly: a breakdown of chiral symmetry leading to non-conservation of chiral charge after the quantization of relativistic particles. The effect was predicted theoretically for Weyl particles, 
and eventually demonstrated in Weyl semimetals. Weyl nodes of opposing chirality are detached in momentum space, and their spins are locked into their momentum directions. When this effect is present, chiral charge appears to no longer be conserved in the presence of parallel electric and magnetic fields. The end result is that a Weyl semimetal is more conductive than a nonmagnetic metal in the presence of an increasing magnetic field.

The anomalous Hall effect (AHE) is another intriguing phenomenon related to Weyl semimetals. This effect describes a contribution to the resistivity that is orthogonal to the current direction, in proportion to the magnetization. It is usually unknown whether this is caused by the crystal structure (intrinsic AHE) or by an impurity in the material (extrinsic AHE). In general, both types of AHE can be present and are of the same order of magnitude, making it very challenging to separate them experimentally. However, AHE is common in ferromagnetic Weyl semimetals. In this subclass, the appearance of nodes is related to the breaking of time reversal symmetry [16]. The extrinsic AHE is negligible in these materials, so the AHE has a purely intrinsic origin. Furthermore, the intrinsic AHE is fully determined by the relative positions and topological charges of the Weyl nodes.

It is very intriguing how quantum effects combine with magnetic phenomena in magnetic semimetals. For example, it has been shown that a Fe3Sn2 [17] material supports bulk massive Dirac fermions in the presence of ferromagnetic order or a half-metallic ferromagnetic material (e.g., Co3Sn2S2 [18]) with magnetic Weyl fermions. In addition, a small amount of AHE conductivity has been measured in Co2TiGe thin films [19] and in recently discovered topological magnetism in the predicted magnetic Weyl semimetal CeAlGe [20].

In ferromagnetic materials, the domain structure of ferromagnetic materials is an interesting research subject, and this structure has an important role in energy density parameters like coercive force and internal magnetization. It is therefore highly desirable to build experimental systems that can simultaneously study the magnetic and quantum properties of magnetic Weyl semimetals.

The most extensive system for studying quantum phenomena is angle-resolved photoemission spectroscopy (ARPES) [21-26]. This method is also used to study magnetic semimetals [27,28] as well as to perform time-resolved [29] and spin-resolved [30] photoemission experiments. ARPES is a powerful experimental technique that provides information regarding the energy and momentum of the electronic state. However, besides high-resolution ARPES images in the case of spin- and time-resolved images, this technique is usually limited to large or epitaxial samples [31].

An easier way to achieve the necessary magnetic information of magnetic Weyl semimetals is by magneto-optical methods. For example, information about the internal magnetization and anisotropy of Co2MnGa thin films [32] or learning about the domain structures of Mn3Sn thin films can be carried out by performing magneto-optical imaging [33]. Magneto-optical methods are based on the polarization rotation of the light interacting with the material, which can be the rotation of transmitted or reflected light. It is called the magneto-optical Faraday (or simply Faraday) effect for transmitted light or the magneto-optical Kerr effect (MOKE) for reflected light. The Faraday effect is used for transparent materials. If a sample is placed on a substrate, the MOKE is more suitable. The MOKE and the Faraday effect occur due to the existence of off-diagonal elements in a dielectric tensor, which depends on the magnetic field. The MOKE has different configurations that can measure different parameters. The types of MOKE configurations are explained in the Materials and Methods section.

In the cases where it is technically difficult to perform photoemission experiments, like in non-epitaxial substrates, the magneto-optical methods can give unique information. The MOKE technique gives an opportunity to study the noncontact effects in magnetic materials, having off-diagonal elements of the dielectric tensor depending on magnetization or an external magnetic field. Different parameters can be changed in magneto-optical Kerr measurements, and this method can also be combined with other techniques. For example, Zhang et al. [34] showed that the chiral anomaly measured in the AHE, together with the Kerr effect, led to measuring the effect in parallel with the magnetic and electric fields. In addition, Yeats et al. [35] showed that exerting dynamic optical control over the magnetization and chemical potentials of a topological insulator 
could help characterize the edge states expected at magnetic domain walls in quantum anomalous Hall insulators. Moreover, Jadidi et al. [36] demonstrated chiral control of optical laser pulse pump-probe measurements. These types of experiments with MOKE measurements were performed on ferromagnetic materials [37-46]. In all of these experiments, the results were achieved by following magneto-optical signal dynamics. Due to the fact that the magnetic response depends not only on an external magnetic field but also on internal magnetization, hysteresis loops are achieved. The response and magnetic dynamics of the systems describe a coercive field and the spontaneous magnetization of materials. Using time-resolved magneto-optical experiments, it is possible to change the wavelengths as well as the intensities of the pumping or probing laser pulses. In order to perform spatial-resolved measurements in addition to time-resolved measurements, Kerr imaging measurements can be carried out.

It is possible to follow the dynamics of the processes by performing time-resolved laser pulse pump probe experiments. Using this technique, the dynamics of the Kerr signal can be followed, and it is possible to study the dynamic behavior of different degrees of freedom like electrons, spins, and lattice over time as well as the interactions between them. In several cases, this signal does not come from magnetization, but from the electron redistribution over its energy levels. For this purpose, it uses an additional parameter such as pump energy density, which separates between real magnetization and redistribution of electrons over its energy levels (band-filling effect). The pioneering work of Beaurepaire et al. [45] revealed that a 40-nm thin nickel film can be demagnetized in $100 \mathrm{fs}$. In addition, Koopmans et al. [37] proposed that this is possible due to band-filling effects. Cheskis et al. [46] showed that at a high laser pulse excitation saturates the magneto-optical signal. Additional information regarding the material can be achieved using a MOKE imaging system instead of collecting information from a single detector that uses an imaging camera. Furthermore, Elasar et al. [47] demonstrated that it is possible to measure magnetic reversal using single-pulse excitation.

In this review, we illustrate many experimental possibilities described in the literature. First, we describe the magneto-optical measurements of chiral anomaly using the Kerr effect of the rotated magnetic field, where rotation of the magnetic field is a variable. Then, we explain experiments in which the magneto-optical signal is measured together with the AHE as well as ultrafast magnetic dynamics showing variation of the pumping laser pulse intensity. Finally, we describe the magneto-optical Kerr and Faraday imaging systems.

\section{Materials and Methods}

Magneto-optical methods consist of the rotation of polarized laser light. In order to measure this rotation, two linear polarizers are placed before and after the sample to be measured. These polarizers are placed across from each other. After the second analyzer, a single detector or a microscope and a charge-coupled device (CCD) camera is situated to make a single detection or to take an image, respectively. This setup is used for reflected light (MOKE) or transmitted (Faraday) light. For MOKE, three setups are possible: polar, longitudinal, and transverse. Each is appropriate for a specific situation, but together, they cause off-diagonal elements of the dielectric tensor.

For chiral anomaly, the angle between the electric field that causes the electric current and the magnetic field is defined as the Kerr rotation. This rotation can change with the value of the current or with the angle between the two fields. Thus, the angle that is measured depends on the rotation of the magnetic field relative to the electric field.

Another method combines the measurement of the AHE and the MOKE. The AHE system uses the usual Hall system voltage that is measured orthogonally to the current direction and the magnetic field that is orthogonal to both of them. This Hall system, combined with a single-detector MOKE system, is able to provide all required information.

Following the MOKE measurements, it is possible to determine the history of the magnetic field and coercivity. It is accepted that the Kerr rotation measures the changing external magnetic field in the closed loop. 
With Faraday or Kerr imaging system measurements, we can include the dependence of measurements on the time interval between a pair of pulses. In this way, it is possible to follow the magnetic dynamics inside the material. Two additional parameters are the direction and the type of light polarization. In this review, we describe the experiments that use all of these types of measurements. First, the MOKE rotational measurement that combines the electro transport setup was used in order to measure the chiral anomaly. These measurements from the magneto-optical system require only the measurement of rotation of the reflected signal. The other system shows not only rotation, but also the magnetic history. The simultaneous response of the anomalous Hall transport and the MOKE systems are shown. Furthermore, regarding the magnetic history and domains, the further system describes the intensity of the excitation as a significant parameter in MOKE measurements. The first part pronounces single-detector measurements done on 30-nm polycrystalline nickel. The magnetic dynamics are pronounced as the time delay between the probe and pump laser pulses. The second part is devoted to the MOKE imaging system where the Kerr imaging technique is needed to define spatial dynamics after magnetic reversal and domain wall movement. Additionally, we describe a complete experimental program on the magneto-optical responses of nickel in the picosecond regime, with respect to variations in excitation density. By using time-resolved MOKE measurements to follow the apparent magnetization and coercivity, this study demonstrates a drop in magneto-optical contrast at short time intervals. Furthermore, a saturation of this effect was observed at high excitation densities, even as the temperature reached by the sample kept increasing. The only plausible reason for these observations is the band-filling effect. This explanation is bolstered by a quantitative comparison of excitation densities to known band structure and by the specific heat data of nickel. Hence, we associate the saturation of magneto-optical contrast during electronic thermalization with the full collapse of the Stoner gap. We also observed that the remnant magnetization persisted at high excitation densities, and from this, we can conclude that spin relaxation is incomplete over the measured time scale. In this paper, we demonstrated time-resolved measurements on 30-nm nickel. Nucleation of the opposite polarization conducted by single-pulse-excited magnetic reversal is also shown.

\section{Results}

\subsection{Kerr Rotation as a Function of the Electric and Magnetic Fields}

The experimental system is shown in Figure 1a [34]. The magnetic field, B, can rotate in the $\mathrm{x}-\mathrm{Z}$ plane, and a constant electric current flows in the same plane. In the experiment, we used a horizontally polarized laser with a wavelength of $670 \mathrm{~nm}$. The effect of the chiral anomaly was measured as a function of the current and the angle between the electric and magnetic fields. The magnetic field was rotated relative to the electric field while the polar MOKE system measured the Kerr rotation. The resulting rotational magneto-optical Kerr effect (ROT-MOKE) data on bulk $\mathrm{Cd}_{3} \mathrm{As}_{2}$ single crystals are shown in Figure 1b. No Kerr signals from the crystal surface were detected for a weak magnetic field (up to 2000 Gauss), as predicted for a system with both inversion and time-reversal symmetries intact. However, when a direct-current electric bias was applied across the sample, we observed impressively large Kerr rotation signals with increasing current density (from 0 to $144 \mathrm{~mA} \cdot \mathrm{mm}^{-2}$ ), as seen in Figure 1b. The ROT-MOKE curves are proportional to the cosine of the angle between the $\mathrm{E}$ and $\mathrm{B}$ fields: its absolute value reaches a maximum when they are parallel $\left(0^{\circ}\right)$ and fell to zero when they were perpendicular $\left(90^{\circ}\right)$. This behavior confirmed our expectation that the complex Hall conductivity of a gyrotropic tensor and the breaking of time-reversal symmetry cause the Kerr effect [48]. However, it is not the only explanation: alternatively, the Kerr effect could be caused by spin polarization provoked by the E and B fields. Indeed, the chiral anomaly causes the Weyl nodes to polarize. Charge transfer between a pair of Weyl nodes takes place across the surface of Fermi arcs, which are known to have a high degree of spin polarization as verified by recent photoemission studies $[49,50]$. 
a

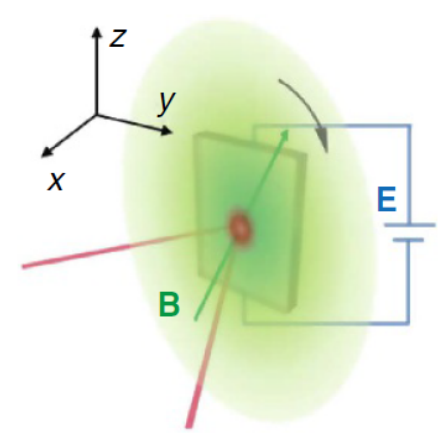

b

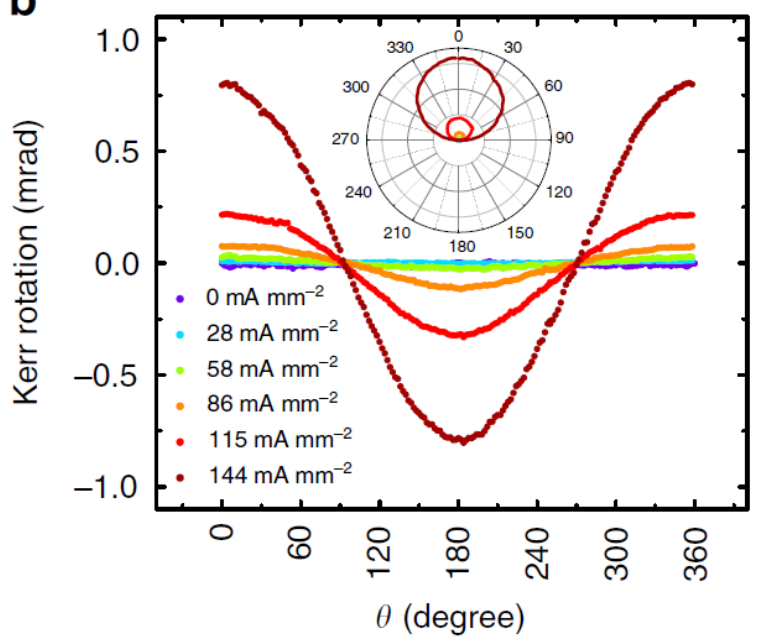

Figure 1. (a) The experimental MOKE setup and (b) the rotational MOKE data of $\mathrm{Cd}_{3} \mathrm{As}_{2}$ bulk crystals. Magnetic field rotation in the $y-z$ plane is parallel to the sample surface. The input laser is $p$-polarized, with a wavelength of $670 \mathrm{~nm}$. The rotational MOKE signals of the $\mathrm{Cd}_{3} \mathrm{As}_{2}$ crystals under different current densities and a constant magnetic field accepted a cosine-function dependence on y. Here, y is defined as the angle between E and B. The magnetic field was fixed at 2000 Oe (from [34]).

\subsection{Magneto-Optical Kerr Signal Combined with Anomalous Hall Effect Measurements}

This experiment, as shown in Figure 2, performed simultaneous magneto-optical and magneto-transport measurements to describe the ferromagnetism of the samples. We used thin films of $\mathrm{Cr}-(\mathrm{Bi}, \mathrm{Sb})_{2} \mathrm{Te}_{3}$ cooled to $\mathrm{T}=2.9 \mathrm{~K}$. Figure $2 \mathrm{~A}$ demonstrates the development of the polar Kerr angle, $\theta_{\mathrm{K}}$, and the anomalous Hall resistance $R_{x y}$. These data were taken from a typical five-layer region of the $\mathrm{Cr}-(\mathrm{Bi}, \mathrm{Sb})_{2} \mathrm{Te}_{3}$ film. The two series are shown as functions of the magnetic field strength (increasing or decreasing) [35]. A roughly square hysteresis was clearly visible in the MOKE and AHE curves, representing ferromagnetic ordering with an easy axis perpendicular to the sample plane. It was considered that the film had a Curie temperature. Figure $2 \mathrm{~B}$ demonstrates the measurement geometry at $\mathrm{T}_{\mathrm{c}} \approx 18 \mathrm{~K}$, while Figure $2 \mathrm{C}$ shows the longitudinal resistance $R_{x x}$ as a function of the applied field at $2.9 \mathrm{~K}$. Peaks at $\pm H_{c}$ have been observed in comparable materials and are recognized to increase scattering due to magnetic disorder during coercion [28]. Although other films with the same composition, grown in the same molecular-beam epitaxy chamber, have previously demonstrated quantized conductance at $\mathrm{T}=1 \mathrm{~K}$, the absolute resistances measured here show that at $\mathrm{T}>2.9 \mathrm{~K}$, the conductivity is dominated by surface and bulk channels rather than quantized edge states. During the MOKE experiments, optical illumination can also affect the magnetization of the film. Figure 2D shows the coercive field $H_{c}$ as a function of the laser power (spot size $\sim 1 \mu \mathrm{m}, \mathrm{T}=3.2 \mathrm{~K}$ ). These values are derived from the MOKE hysteresis measurements. We can see that the coercivity decreases with illumination between $20 \mu \mathrm{W}$ and $2 \mathrm{~mW}$, where local heating is likely to dominate. The trend is well fit by a power law, but plateaus at low powers to a value that agrees with the coercivity extracted from the AHE hysteresis without illumination (red arrow). This suggests that during MOKE measurements, a level of optical illumination below $2 \mu \mathrm{W}$ has little effect on the magnetic properties of the film. 

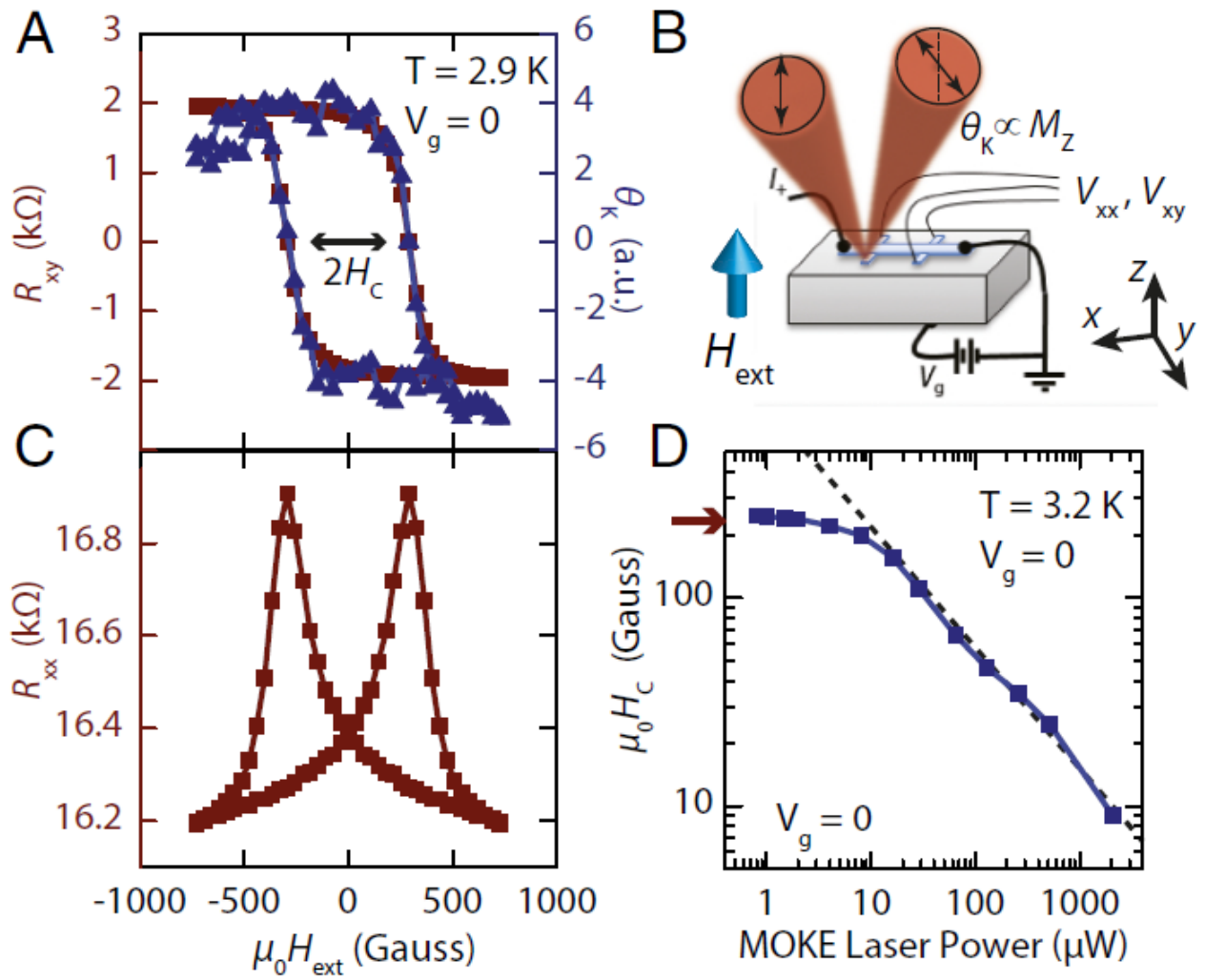

Figure 2. Synchronized MOKE and $\mathrm{AHE}$ measurements in a film of $\mathrm{Cr}-(\mathrm{Bi}, \mathrm{Sb})_{2} \mathrm{Te}_{3}$ at $\mathrm{T}=2.9 \mathrm{~K}$ (from [35]). (A) Magnetic hysteresis loops presenting an anomalous Hall resistance $R_{x y}$ (red squares) and a Kerr angle $\theta_{\mathrm{K}}$ (blue triangles). They depend on the magnetic field strength. MOKE was done with a spot size of $\approx 1 \mu \mathrm{m}$ and a laser power of $\approx 1 \mu \mathrm{W}$. (B) Diagram of the experimental setup. MOKE measurements were made on the unused leg of the Hall bar to avoid optical effects on transport. (C) Magnetic hysteresis with a longitudinal resistance $R_{x x}$. (D) Coercive field $H_{C}$ of the film from MOKE hysteresis measurements as a function of the MOKE laser power. The red arrow specifies the $H_{C}$ value obtained from AHE hysteresis without illumination (from [35]).

\subsection{Magneto-Optical Kerr Signal as a Function of Pump Intensity}

The experiment demonstrated in Figure 3a shows values of $M_{s}$ obtained from a large set of hysteresis curves, measured as a function of laser energy density and pulse time delay. All sample films were grown on glass substrates [46]. The data for various time delays (100 fs, $150 \mathrm{fs}, 300 \mathrm{fs}$, 1 ps, and 3 ps) were all normalized to $M_{0}$, which is the value of $M$ obtained when the pump beam is blocked. These were plotted against the energy density of the beam. A typical error bar is displayed beside the data rather than drawing similar bars on all points. At first glance, the data appear noisy, but close inspection discloses some consistent trends. In particular, there was a clear difference between thetemporal behaviors seen at low beam energy densities $\left(<20 \mathrm{~mJ} / \mathrm{cm}^{2}\right)$ and high pump energy densities $\left(>35 \mathrm{~mJ} / \mathrm{cm}^{2}\right)$ : at low energy densities, the MOKE response reached a maximum around $300 \mathrm{fs}$ just after the excitation ended, and slowly decayed for longer time delays (1-3 ps). As an alternative, at high energy densities, the MOKE response continued to rise long after the excitation was over. Note that at early times, the values of $M s$ were equal, and the change in behavior happened at the same excitation density. It is important to note that these observations are in excellent agreement with the behavior of the differential reflection with increasing excitation density. As presented in Figure $3 b$, the rising differential reflection signal continues toward longer time delays as the pump energy density rises. This correlation between the magnetic MOKE signal and nonmagnetic differential reflection signal suggests that the measured dynamics of the former are not entirely magnetic in origin. Moreover, 
this observation permits the lines to be drawn that assist as directors in Figure $3 \mathrm{a}$ and demonstrate the general trends outlined above.

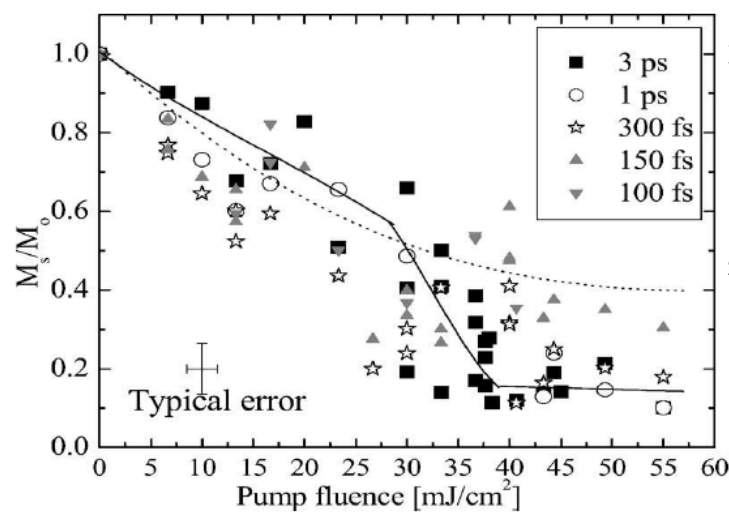

(a)

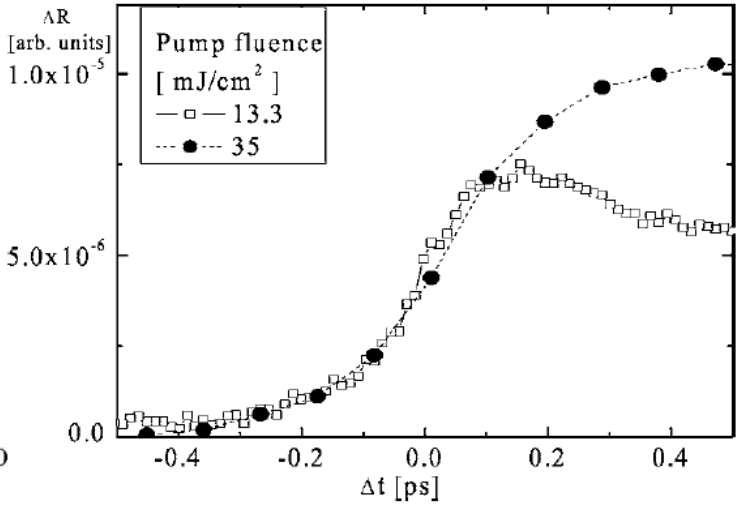

(b)

Figure 3. (a) The normalized spontaneous magnetization in response to the laser energy density for few time delays in the picosecond and subpicosecond range. (b) The differential reflection signal as function of the time delay for two pump energy densities: $13.3 \mathrm{~mJ} / \mathrm{cm}^{2}$ and $35 \mathrm{~mJ} / \mathrm{cm}^{2}$ (from [46]).

The most noteworthy feature of Figure 3 is the clear saturation of the MOKE response at high pump energy densities. While the magneto-optical difference gradually declined with longer time intervals, there was no apparent reduction as the pump energy density increased past $35 \mathrm{~mJ} / \mathrm{cm}^{2}$ for any time interval. For all measurements in the picosecond and sub-ps ranges, a residual magneto-optical difference was shown at the maximum possible pump energy density. The saturation of the MOKE response at high pump energy densities is shown in more detail in Figure 3.

\subsection{Magneto-Optical Kerr and Faraday Imaging}

Here, we elucidate a magneto-optical imaging system (Figure 4a) that combines single-pulse measurements with $100 \mathrm{fs}$ temporal resolution and up to $\sim 50 \mu \mathrm{m}$ spatial resolution, consequently permitting exploration of the reversal dynamics in detail [47]. A Ti:sapphire regenerative amplifier ( $70 \mathrm{fs}, 1 \mathrm{kHz}$ ) and a slightly modified time-resolved pump-probe setup were used so that the probe spot size covered a large area of the sample. This setup greatly increases the area excited by the pump beam. Hence, the probe pulse provides a 70-fs illumination (or "flash"). The magnetization measurements require the MOKE to use conventional optical polarizers. The sample is depicted inside the coils of an electromagnet. The magnetic field strength is controlled via the current provided by its power supply. A lens with a focal length of $75 \mathrm{~mm}$ was used to image the sample on a Si CCD camera, with $1024 \times 128$ pixels. Each pixel was $26 \mu \mathrm{m} \times 26 \mu \mathrm{m}$ in size, and the magnification factor was 10x. To limit scattering from the pump beam onto CCD pixels, we have the option of introducing an iris behind the lens, although this also decreases the numerical aperture and the spatial resolution. Each CCD image characterizes the spatial distribution of the transmitted probe. To recover the spatial magnetization distribution, a reference image (i.e., a negative field) is subtracted from the data. 


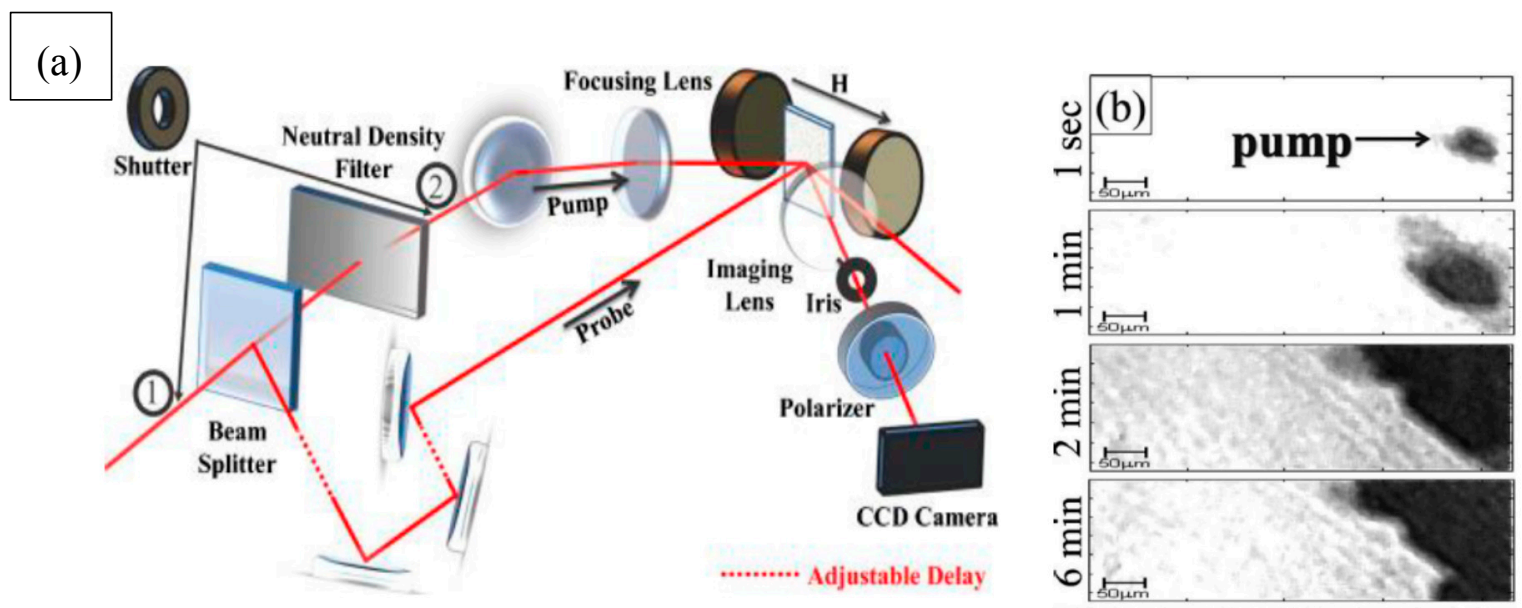

Figure 4. (a) Schematic of the time-resolved MOKE microscope. The numbers " 1 " and " 2 " represent the alternative shutter positions for a fast pump-probe. (b) The magnetic reversal in a permalloy sample (from [47]).

Figure 4 exhibits two examples of nonlocal effects arising from a single pulse. As shown in Figure $4 \mathrm{~b}$, the excitation area falls inside a "soft" region, demonstrating that the pump-induced reversal is a point of nucleation. The reversal propagates away from this point, eventually covering the same area of the sample that would have instead reversed spontaneously. The sample achieves a steady state after approximately 3 min.

Figure 5 shows the system used to measure ultrafast magnetization dynamics during the process of all-optical reversal. As mentioned in another study, the measurement was based on single-shot time-resolved Faraday magneto-optical microscopy [51]. The setup had a spatial resolution of $1 \mu \mathrm{m}$. In this study, a single optical pump pulse of 100-2100 fs excited the film (see Figure 5a). A single, lower intensity, 100-fs optical probe pulse was triggered after the pump pulse to capture a magneto-optical image of the sample. In this image, opposing magnetization directions appeared as black and white areas due to the Faraday effect. Mixed magnetic states appeared as shades of gray. Altering the laser pulse energy density $(\mathrm{F})$ changes of the magnetic state observed after the pulse are shown in Figure $5 \mathrm{~b}$. Reversal did not occur for values of $F$ less than about $3.14 \mathrm{~mJ} / \mathrm{cm}^{2}$. Above this fluence, however, we observed purely optical, helicity-dependent reversal. If $\mathrm{F}$ was even a little higher than this threshold value, then magnetization reversal was detected following laser pulses of both helicities, and even a linearly polarized pulse can trigger the reversal. Nevertheless, the size of the reversal area appears to be slightly larger if the helicity of the laser pulse favors the helicity-dependent reversal at a lower energy density. This is shown in the last row in Figure $5 \mathrm{~b}$. At laser energy densities greater than $\sim 4.7 \mathrm{~mJ} / \mathrm{cm}^{2}$, a multi-domain state forms. As demonstrated in Figure 5b, the Gaussian spatial profile of high-energy density laser pulses $\left(\mathrm{F}>4.5 \mathrm{~mJ} / \mathrm{cm}^{2}\right)$ can be separated into four regions, corresponding to the four types of effect that the pulses can have on the magnetic medium: no reversal; helicity-dependent, purely optical reversal; helicity-independent reversal; and the formation of a multi-domain state. Note that the area containing helicity-dependent magnetization reversal is much smaller than the laser spot. 

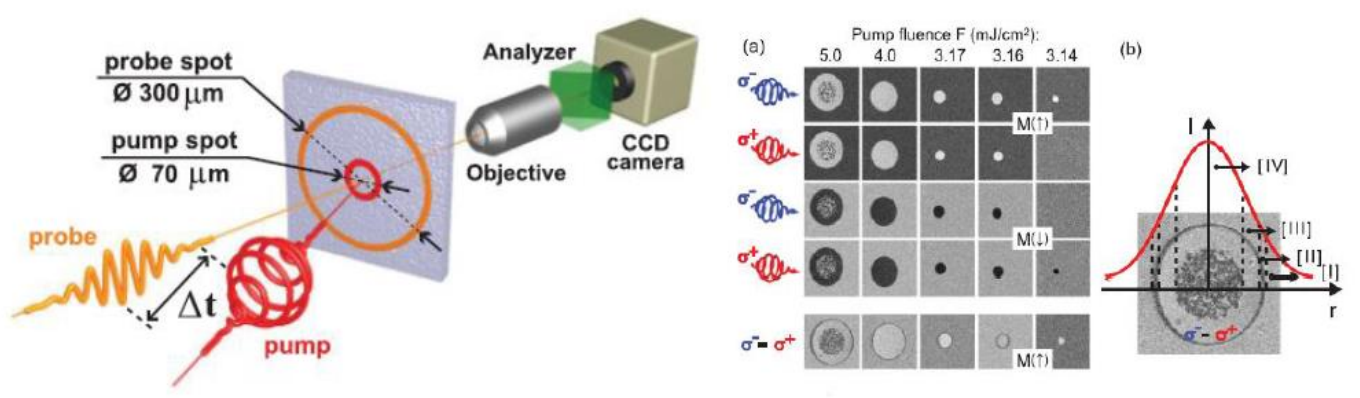

Figure 5. (a) Plan of the single-shot time-resolved Faraday magneto-optical microscopy experiment and (b) steady-state magnetooptical images of the $\mathrm{Gd}_{26} \mathrm{FeCo}$ sample at room temperature. The images were taken after a single 100-fs $\sigma^{+}$- or $\sigma^{-}$-polarized laser pulse with energy density F. Before each pulse, the sample was returned to a single-domain state by applying a uniform magnetic field pulse (from [51]).

\section{Discussion}

In this review, it was shown that magneto-optical tools, like chiral anomaly and AHE combined with other techniques, are essential and uncomplicated ways in which to study the quantum effects in Weyl semimetals. It is possible to study these effects in the steady state with continuous laser light, with pulsed or an ultrafast laser pulse by following the dynamics of the processes. In ferromagnetic Weyl semimetals, it is possible to follow the history of the process by looking at hysteresis and analyzing parameters like coercivity and internal magnetization. It is also possible to perform magnetic imaging using MOKE or Faraday imaging systems. In addition, one more intriguing issue is to implement Floquet-Weyl materials using ultrafast circular polarized pulses [52,53]. They can also take on periodic Floquet-Weyl properties in which the time-dependent Weyl point can be organized. In order to excite the Weyl nodes, it is necessary to use mid-infrared laser pulses. Lately, Ma et al. have demonstrated the direct detection of the chirality of the Weyl fermions using continuous $\mathrm{CO}_{2}$ laser making excitation near the Weyl node energies [54]. Recent experiments have shown that it is possible to produce a femtosecond pulse $<1$ ps until a wavelength of $15 \mu \mathrm{m}$ [55].

MOKE systems are not new. However, new possibilities like a mid-infrared wavelength range that was not possible previously, a high polarization extinction ratio that makes it feasible to detect tiny Kerr rotation [56], much more stable lasers, and very sensitive locks in amplifiers together with all of the experimental experience that has been achieved in magneto-optical studies of ferromagnetic materials will contribute to the subject of the combination of Weyl and magnetic materials, topology, and magnetism.

\section{Conclusions}

In this review, we are reminded that magneto-optical tools are still the best way to study magnetic phenomena and are also relevant to magnetic Weyl semimetals. Moreover, the MOKE technique is also used to study chiral anomaly in nonmagnetic Weyl semimetals. The MOKE and Faraday techniques, particular ultrafast femtosecond techniques, can provide a lot of information in the area of Weyl and Dirac semimetals as well as additional information. All experience that has been achieved regarding ferromagnetic materials, both in single detection and magnetic imaging, not only contributes to knowledge about Dirac and Weyl semimetals, but possibly can add knowledge into the area of Dirac and Weyl fermions in high-energy physics. The new possibility of using mid-infrared femtosecond pulses to excite Floquet-Weyl modes will be a great contribution to this field of research.

Funding: This research received no external funding.

Acknowledgments: The author would like to acknowledge Y. Gorodetski for our fruitful discussions.

Conflicts of Interest: The author declares no conflict of interest. 


\section{References}

1. Geim, A.K.; Novoselov, K.S. The rise of graphene. Nat. Mater. 2007, 6, 183-191. [CrossRef] [PubMed]

2. Hasan, M.Z.; Kane, C.L. Colloquium: Topological insulators. Rev. Mod. Phys. 2010, 82, 3045. [CrossRef]

3. Qi, X.-L.; Zhang, S.-C. Topological insulators and superconductors. Rev. Mod. Phys. 2011, 83, 1057. [CrossRef]

4. Vafek, O.; Vishwanath, A. Dirac fermions in solids: From high-T c cuprates and graphene to topological insulators and Weyl semimetals. Annu. Rev. Condens. Matter Phys. 2014, 5, 83-112. [CrossRef]

5. Jia, S.; Xu, S.-Y.; Hasan, M.Z. Weyl semimetals, Fermi arcs and chiral anomalies. Nat. Mater. 2016, 15, 1140-1144. [CrossRef]

6. Yan, B.; Felser, C. Topological materials: Weyl semimetals. Annu. Rev. Condens. Matter Phys. 2017, 8, 337-354. [CrossRef]

7. Hu, J.; Xu, S.-Y.; Ni, N.; Mao, Z. Transport of topological semimetals. Annu. Rev. Mater. Res. 2019, 49, 207-252. [CrossRef]

8. Armitage, N.P.; Mele, E.J.; Vishwanath, A. Weyl and Dirac semimetals in three-dimensional solids. Rev. Mod. Phys. 2018, 90, 15001. [CrossRef]

9. Bernevig, A.; Weng, H.; Fang, Z.; Dai, X. Recent progress in the study of topological semimetals. J. Phys. Soc. Jpn. 2018, 87, 41001. [CrossRef]

10. Young, S.M.; Zaheer, S.; Teo, J.C.Y.; Kane, C.L.; Mele, E.J.; Rappe, A.M. Dirac semimetal in three dimensions. Phys. Rev. Lett. 2012, 108, 140405. [CrossRef]

11. Wang, Z.; Sun, Y.; Chen, X.-Q.; Franchini, C.; Xu, G.; Weng, H.; Dai, X.; Fang, Z. Dirac semimetal and topological phase transitions in A $3 \mathrm{Bi}(\mathrm{A}=\mathrm{Na}, \mathrm{K}, \mathrm{Rb})$. Phys. Rev. B 2012, 85, 195320. [CrossRef]

12. Wang, Z.; Weng, H.; Wu, Q.; Dai, X.; Fang, Z. Three-dimensional Dirac semimetal and quantum transport in Cd 3 As 2. Phys. Rev. B 2013, 88, 125427. [CrossRef]

13. Xu, S.-Y.; Belopolski, I.; Alidoust, N.; Neupane, M.; Bian, G.; Zhang, C.; Sankar, R.; Chang, G.; Yuan, Z.; Lee, C.-C. Discovery of a Weyl fermion semimetal and topological Fermi arcs. Science 2015, 349, $613-617$. [CrossRef] [PubMed]

14. Dirac, P.A.M. The quantum theory of the electron. Proc. R. Soc. Lond. Ser. A Contain. Pap. Math. Phys. Character 1928, 117, 610-624.

15. Weyl, H. Elektron und gravitation. I. Z. Phys. 1929, 56, 330-352. [CrossRef]

16. Burkov, A.A. Chiral anomaly and diffusive magnetotransport in Weyl metals. Phys. Rev. Lett. 2014, 113, 247203. [CrossRef]

17. Ye, L.; Kang, M.; Liu, J.; Von Cube, F.; Wicker, C.R.; Suzuki, T.; Jozwiak, C.; Bostwick, A.; Rotenberg, E.; Bell, D.C. Massive Dirac fermions in a ferromagnetic kagome metal. Nature 2018, 555, 638-642. [CrossRef]

18. Wang, Q.; Xu, Y.; Lou, R.; Liu, Z.; Li, M.; Huang, Y.; Shen, D.; Weng, H.; Wang, S.; Lei, H. Large intrinsic anomalous Hall effect in half-metallic ferromagnet Co 3 Sn 2 S 2 with magnetic Weyl fermions. Nat. Commun. 2018, 9, 1-8.

19. Dulal, R.P.; Dahal, B.R.; Forbes, A.; Bhattarai, N.; Pegg, I.L.; Philip, J. Weak localization and small anomalous Hall conductivity in ferromagnetic Weyl semimetal Co 2 TiGe. Sci. Rep. 2019, 9, 1-7. [CrossRef]

20. Puphal, P.; Pomjakushin, V.; Kanazawa, N.; Ukleev, V.; Gawryluk, D.J.; Ma, J.; Naamneh, M.; Plumb, N.C.; Keller, L.; Cubitt, R. Topological Magnetic Phase in the Candidate Weyl Semimetal CeAlGe. Phys. Rev. Lett. 2020, 124, 17202. [CrossRef]

21. Chang, C.-Z.; Zhang, J.; Feng, X.; Shen, J.; Zhang, Z.; Guo, M.; Li, K.; Ou, Y.; Wei, P.; Wang, L.-L. Experimental observation of the quantum anomalous Hall effect in a magnetic topological insulator. Science 2013, 340, 167-170. [CrossRef] [PubMed]

22. Bostwick, A.; Ohta, T.; Seyller, T.; Horn, K.; Rotenberg, E. Quasiparticle dynamics in graphene. Nat. Phys. 2007, 3, 36-40. [CrossRef]

23. Sprinkle, M.; Siegel, D.; Hu, Y.; Hicks, J.; Tejeda, A.; Taleb-Ibrahimi, A.; Le Fèvre, P.; Bertran, F.; Vizzini, S.; Enriquez, H. First direct observation of a nearly ideal graphene band structure. Phys. Rev. Lett. 2009, 103, 226803. [CrossRef] [PubMed]

24. Chen, Y.L.; Analytis, J.G.; Chu, J.-H.; Liu, Z.K.; Mo, S.-K.; Qi, X.-L.; Zhang, H.J.; Lu, D.H.; Dai, X.; Fang, Z. Experimental realization of a three-dimensional topological insulator, Bi2Te3. Science 2009, 325, $178-181$. [CrossRef] [PubMed] 
25. Liu, Z.K.; Zhou, B.; Zhang, Y.; Wang, Z.J.; Weng, H.M.; Prabhakaran, D.; Mo, S.-K.; Shen, Z.X.; Fang, Z.; Dai, X. Discovery of a three-dimensional topological Dirac semimetal, Na3Bi. Science 2014, 343, 864-867. [CrossRef] [PubMed]

26. Liu, Z.K.; Jiang, J.; Zhou, B.; Wang, Z.J.; Zhang, Y.; Weng, H.M.; Prabhakaran, D.; Mo, S.K.; Peng, H.; Dudin, P. A stable three-dimensional topological Dirac semimetal Cd 3 As 2. Nat. Mater. 2014, 13, 677-681. [CrossRef] [PubMed]

27. Xu, Q.; Liu, E.; Shi, W.; Muechler, L.; Gayles, J.; Felser, C.; Sun, Y. Topological surface Fermi arcs in the magnetic Weyl semimetal Co 3 Sn 2 S 2. Phys. Rev. B 2018, 97, 235416. [CrossRef]

28. Liu, D.F.; Liang, A.J.; Liu, E.K.; Xu, Q.N.; Li, Y.W.; Chen, C.; Pei, D.; Shi, W.J.; Mo, S.K.; Dudin, P. Magnetic Weyl semimetal phase in a Kagomé crystal. Science 2019, 365, 1282-1285. [CrossRef]

29. Crepaldi, A.; Autès, G.; Gatti, G.; Roth, S.; Sterzi, A.; Manzoni, G.; Zacchigna, M.; Cacho, C.; Chapman, R.T.; Springate, E.; et al. Enhanced ultrafast relaxation rate in the Weyl semimetal phase of $\mathrm{MoTe}_{2}$ measured by time- and angle-resolved photoelectron spectroscopy. Phys. Rev. B 2017, 96, 241408. [CrossRef]

30. Fanciulli, M.; Schusser, J.; Lee, M.-I.; El Youbi, Z.; Heckmann, O.; Richter, M.C.; Cacho, C.; Spezzani, C.; Bresteau, D.; Hergott, J.-F.; et al. Spin, time, and angle resolved photoemission spectroscopy on $\mathrm{WTe}_{2}$. Phys. Rev. Res. 2020, 2, 13261. [CrossRef]

31. Cattelan, M.; Fox, N.A. A perspective on the application of spatially resolved ARPES for $2 \mathrm{D}$ materials. Nanomaterials 2018, 8, 284. [CrossRef]

32. Finizio, S.; Kronenberg, A.; Vafaee, M.; Foerster, M.; Litzius, K.; de Lucia, A.; Menteş, T.O.; Aballe, L.; Krüger, B.; Jourdan, M. Magnetic configurations in nanostructured Co2MnGa thin film elements. New J. Phys. 2015, 17, 83030. [CrossRef]

33. Higo, T.; Man, H.; Gopman, D.B.; Wu, L.; Koretsune, T.; van't Erve, O.M.J.; Kabanov, Y.P.; Rees, D.; Li, Y.; Suzuki, M.-T. Large magneto-optical Kerr effect and imaging of magnetic octupole domains in an antiferromagnetic metal. Nat. Photonics 2018, 12, 73. [CrossRef] [PubMed]

34. Zhang, C.; Zhang, E.; Wang, W.; Liu, Y.; Chen, Z.-G.; Lu, S.; Liang, S.; Cao, J.; Yuan, X.; Tang, L. Room-temperature chiral charge pumping in Dirac semimetals. Nat. Commun. 2017, 8, 1-9. [CrossRef]

35. Yeats, A.L.; Mintun, P.J.; Pan, Y.; Richardella, A.; Buckley, B.B.; Samarth, N.; Awschalom, D.D. Local optical control of ferromagnetism and chemical potential in a topological insulator. Proc. Natl. Acad. Sci. USA 2017, 114, 10379-10383. [CrossRef] [PubMed]

36. Jadidi, M.; Kargarian, M.; Mittendorff, M.; Aytac, Y.; Shen, B.; König-Otto, J.; Winnerl, S.; Ni, N.; Murphy, T.E.; Drew, H.D. Optical Control of Chiral Charge Pumping in a Topologcial Weyl Semimetal. Bull. Am. Phys. Soc. 2019, 64, po4.00001.

37. Koopmans, B.; Van Kampen, M.; Kohlhepp, J.T.; De Jonge, W.J.M. Ultrafast magneto-optics in nickel: Magnetism or optics? Phys. Rev. Lett. 2000, 85, 844. [CrossRef]

38. Zhang, G.P.; Hübner, W.; Lefkidis, G.; Bai, Y.; George, T.F. Paradigm of the time-resolved magneto-optical Kerr effect for femtosecond magnetism. Nat. Phys. 2009, 5, 499-502. [CrossRef]

39. Regensburger, H.; Vollmer, R.; Kirschner, J. Time-resolved magnetization-induced second-harmonic generation from the Ni (110) surface. Phys. Rev. B 2000, 61, 14716. [CrossRef]

40. Kampfrath, T.; Ulbrich, R.G.; Leuenberger, F.; Münzenberg, M.; Sass, B.; Felsch, W. Ultrafast magneto-optical response of iron thin films. Phys. Rev. B 2002, 65, 104429. [CrossRef]

41. Van Kampen, M.; Koopmans, B.; Kohlhepp, J.T.; De Jonge, W.J.M. Laser-induced precession in canted-spin ferromagnets. J. Magn. Magn. Mater. 2002, 240, 291-293. [CrossRef]

42. Vodungbo, B.; Gautier, J.; Lambert, G.; Sardinha, A.B.; Lozano, M.; Sebban, S.; Ducousso, M.; Boutu, W.; Li, K.; Tudu, B. Laser-induced ultrafast demagnetization in the presence of a nanoscale magnetic domain network. Nat. Commun. 2012, 3, 1-6. [CrossRef]

43. Deb, M.; Molho, P.; Barbara, B.; Bigot, J.-Y. Controlling laser-induced magnetization reversal dynamics in a rare-earth iron garnet across the magnetization compensation point. Phys. Rev. B 2018, 97, 134419. [CrossRef]

44. Schellekens, A.J.; De Vries, N.; Lucassen, J.; Koopmans, B. Exploring laser-induced interlayer spin transfer by an all-optical method. Phys. Rev. B 2014, 90, 104429. [CrossRef]

45. Beaurepaire, E.; Merle, J.-C.; Daunois, A.; Bigot, J.-Y. Ultrafast spin dynamics in ferromagnetic nickel. Phys. Rev. Lett. 1996, 76, 4250. [CrossRef]

46. Cheskis, D.; Porat, A.; Szapiro, L.; Potashnik, O.; Bar-Ad, S. Saturation of the ultrafast laser-induced demagnetization in nickel. Phys. Rev. B Condens. Matter Mater. Phys. 2005, 72, 014437. [CrossRef] 
47. Elazar, M.; Sahaf, M.; Szapiro, L.; Cheskis, D.; Bar-Ad, S. Single-pulse magneto-optic microscopy: A new tool for studying optically induced magnetization reversals. Opt. Lett. 2008, 33, 2734-2736. [CrossRef] [PubMed]

48. Yuan, X.; Zhang, C.; Zhang, Y.; Yan, Z.; Lyu, T.; Zhang, M.; Li, Z.; Song, C.; Zhao, M.; Leng, P.; et al. The discovery of dynamic chiral anomaly in a Weyl semimetal NbAs. Nat. Commun. 2020, 11, 1259. [CrossRef]

49. Xu, S.-Y.; Belopolski, I.; Sanchez, D.S.; Neupane, M.; Chang, G.; Yaji, K.; Yuan, Z.; Zhang, C.; Kuroda, K.; Bian, G. Spin polarization and texture of the Fermi arcs in the Weyl fermion semimetal TaAs. Phys. Rev. Lett. 2016, 116, 96801. [CrossRef]

50. Lv, B.Q.; Muff, S.; Qian, T.; Song, Z.D.; Nie, S.M.; Xu, N.; Richard, P.; Matt, C.E.; Plumb, N.C.; Zhao, L.X. Observation of Fermi-arc spin texture in TaAs. Phys. Rev. Lett. 2015, 115, 217601. [CrossRef] [PubMed]

51. Vahaplar, K.; Kalashnikova, A.M.; Kimel, A.V.; Gerlach, S.; Hinzke, D.; Nowak, U.; Chantrell, R.; Tsukamoto, A.; Itoh, A.; Kirilyuk, A. All-optical magnetization reversal by circularly polarized laser pulses: Experiment and multiscale modeling. Phys. Rev. B 2012, 85, 104402. [CrossRef]

52. Bucciantini, L.; Roy, S.; Kitamura, S.; Oka, T. Emergent Weyl nodes and Fermi arcs in a Floquet Weyl semimetal. Phys. Rev. B 2017, 96, 41126. [CrossRef]

53. Hübener, H.; Sentef, M.A.; De Giovannini, U.; Kemper, A.F.; Rubio, A. Creating stable Floquet-Weyl semimetals by laser-driving of 3D Dirac materials. Nat. Commun. 2017, 8, 1-8. [CrossRef] [PubMed]

54. Ma, Q.; Xu, S.-Y.; Chan, C.-K.; Zhang, C.-L.; Chang, G.; Lin, Y.; Xie, W.; Palacios, T.; Lin, H.; Jia, S. Direct optical detection of Weyl fermion chirality in a topological semimetal. Nat. Phys. 2017, 13, 842-847. [CrossRef]

55. Tancin, R.J.; Chang, Z.; Gu, M.; Radhakrishna, V.; Lucht, R.P.; Goldenstein, C.S. Ultrafast laser-absorption spectroscopy for single-shot, mid-infrared measurements of temperature, $\mathrm{CO}$, and $\mathrm{CH}_{4}$ in flames. Opt. Lett. 2020, 45, 583-586. [CrossRef]

56. Stamm, C.; Murer, C.; Berritta, M.; Feng, J.; Gabureac, M.; Oppeneer, P.M.; Gambardella, P. Magneto-optical detection of the spin Hall effect in Pt and W thin films. Phys. Rev. Lett. 2017, 119, 87203. [CrossRef] [PubMed]

(C) 2020 by the author. Licensee MDPI, Basel, Switzerland. This article is an open access article distributed under the terms and conditions of the Creative Commons Attribution (CC BY) license (http://creativecommons.org/licenses/by/4.0/). 